\title{
Connaissance du monde économique et relations école - entreprise Les apports d'une étude des représentations sociales sur l'entreprise
}

\section{Anne-Marie Manville et Claude Fabre}

\section{(2) OpenEdition \\ Journals \\ Édition électronique \\ URL : http://journals.openedition.org/trema/1398 \\ DOI : 10.4000/trema. 1398 \\ ISSN : 2107-0997 \\ Éditeur \\ Faculté d'Éducation de l'université de Montpellier}

Édition imprimée

Date de publication : 1 janvier 2003

Pagination : 79-94

ISSN : 1167-315X

\section{Référence électronique}

Anne-Marie Manville et Claude Fabre, « Connaissance du monde économique et relations école entreprise Les apports d'une étude des représentations sociales sur l'entreprise », Tréma [En ligne], 20-21 | 2003, mis en ligne le 01 avril 2003, consulté le 19 avril 2019. URL : http://

journals.openedition.org/trema/1398; DOI : 10.4000/trema.1398

Ce document a été généré automatiquement le 19 avril 2019.

Trema 


\title{
Connaissance du monde économique et relations école - entreprise Les apports d'une étude des représentations sociales sur l'entreprise
}

\author{
Anne-Marie Manville et Claude Fabre
}

L'entreprise reste aujourd'hui le lieu principal d'insertion professionnelle des élèves. C'est pourquoi différents dispositifs ont été mis en place, dans les filières professionnelles, technologiques, et générales, pour favoriser la découverte des entreprises et des métiers, et la connaissance des filières d'orientation et de formation. C'est le cas notamment de la semaine obligatoire de découverte des entreprises pour les élèves de Troisième, et des journées « carrefour des métiers » organisées en collège et en lycée. Propres aux filières professionnelles, les périodes de formation en entreprise permettent également d'appliquer les connaissances acquises et de développer les compétences professionnelles des stagiaires. On constate toutefois que les relations école - entreprise varient assez sensiblement d'un établissement à l'autre, tant au niveau des actions réalisées que de leur imbrication dans un projet plus général. Précisons d'emblée que nous ne défendons pas l'idée d'un partenariat systématique et sans limites entre école et entreprise, pas plus d'ailleurs que celle d'une "professionnalisation» trop précoce de l'élève. Nous sommes toutefois convaincus qu'une meilleure connaissance des entreprises et des milieux professionnels peut contribuer à renforcer l'autonomie de l'élève dans ses choix d'orientation et de formation. Les résultats d'une étude exploratoire des représentations sociales sur l'entreprise réalisée en 2000-2001 auprès de 624 élèves et étudiants de filières tertiaires et 160 professeurs et CPE stagiaires de l'académie de Montpellier fournissent un certain nombre de pistes, d'une part pour expliquer l'état des liens école - entreprise aujourd'hui, et d'autre part pour préciser les axes à privilégier dans l'organisation d'actions en relation avec l'entreprise. 


\section{La mission éducative de l'école : quelle attitude et quelles relations avec les entreprises?}

2 Dans un contexte marqué par de fortes mutations de l'environnement professionnel et des formes d'organisation du travail, le sens de l'école passe pour l'élève et sa famille par la construction conjointe d'apprentissages et d'un devenir, c'est-à-dire à terme d'une insertion sociale et économique. Au contact quotidien de la classe, les enseignants sont ainsi de plus en plus sollicités par les élèves et leurs parents par des demandes d'information et de conseil en orientation. Car choisir son devenir ne va pas de soi, choisir s'apprend. Dans ce rôle d'accompagnateur de la construction de parcours, la plupart des enseignants se trouvent démunis par leur faible connaissance ou leur méconnaissance des organisations du travail, des réalités des milieux professionnels, des formations, des sources documentaires et des méthodes. Dans cette perspective, la rénovation récente de la formation IUFM ${ }^{1}$ des professeurs stagiaires de lycée et collège ${ }^{2}$ et des CPE stagiaires insiste sur la dimension éducative du métier d'enseignant et sur son rôle dans l'accompagnement du parcours de l'élève.

3 Il s'agit en particulier de renforcer la connaissance du monde économique (notamment au travers des stages en entreprise ${ }^{3}$ ) et de sensibiliser les futurs enseignants à la problématique de l'orientation. L'éducation à l'orientation est en effet devenue obligatoire dans les lycées et les collèges ${ }^{4}$ : visant à développer la connaissance des métiers, des entreprises, et des filières de formation, la démarche implique normalement les différents acteurs de l'établissement et les membres de l'équipe pédagogique. De nombreuses actions contribuant à développer l'information sur les entreprise et les métiers sont ainsi organisées dans les collèges et les lycées: visites d'entreprises, interventions de responsables d'entreprises devant les classes, interventions de parents présentant leurs métiers, journées « forum » ou « carrefour » des métiers... L'exploitation de la semaine de découverte de l'entreprise réalisée par les élèves de classe de Troisième peut aussi contribuer à la réflexion sur l'orientation.

4 Au-delà de l'information et de l'aide à l'orientation, on observe des efforts croissants de rapprochements, sous diverses formes et à différents niveaux: stages pour les enseignants, jumelages écoles - entreprises ou classes - entreprises, formations en alternance, conventions entre le Ministère de l'Éducation Nationale et fédérations professionnelles ou des grandes entreprises ${ }^{5}$, etc... De nombreux dispositifs comme les stages, ou, plus récemment, les Projets Pluridisciplinaires à Caractère Pédagogique (PPCP) sont l'occasion de découvrir l'entreprise, d'avoir un premier contact ou une première expérience. Lors du colloque de Lille, en septembre 1999, le Ministre Claude Allègre rappelait les enjeux du rapprochement école-entreprise. Ainsi la Charte des Lycées prévoit-elle la nomination de délégués aux entreprises dans les rectorats. Dans la même optique, la nomination d'Ingénieurs Pour l'École (I.P.E.) ${ }^{6}$ marque la volonté d'une plus grande ouverture sur le monde économique. À terme, la mise en place de dispositifs de stages en entreprise pour les enseignants de toutes filières n'est plus impensable ${ }^{7}$.

On constate néanmoins que la nature des dispositifs et des expérimentations mises en œuvre, la fréquence, l'organisation et l'articulation des différentes actions peuvent varier sensiblement d'un établissement à l'autre, voire d'une académie à l'autre. Si les collaborations et les partenariats sont fréquents dans les formations dites technologiques ou industrielles ${ }^{8}$, les relations entre l'école et l'entreprise posent encore problème dans 
les filières générales. Logiques différentes, valeurs différentes et conceptions différentes de la formation expliqueraient la dissociation entre sphère éducative et sphère professionnelle. L'accumulation des dispositifs de rapprochement conduit, sinon à les discréditer, à s'interroger quant à leur impact réel. Un article de la Lettre de l'Éducation ${ }^{9}$ montre que les liens de partenariat sont limités à quelques initiatives locales: «traditionnelle dans l'enseignement professionnel, la participation des entreprises à la formation des jeunes l'est beaucoup moins dans les voies technologiques, ou, plus encore, générales [...]. Audelà du stage, les partenariats plus élaborés sont rares ». Anne Gonnin-Bolo ${ }^{10}$ souligne que les liens avec l'entreprise sont souvent assurés par le chef d'établissement lui-même ou par le chef des travaux dans les lycées professionnels. S'il est difficile de faire un bilan chiffré, on peut constater que les pratiques sont très variables, et que l'efficacité des dispositifs existants repose davantage sur les personnes que sur les outils proprement dits.

Les relations école - entreprise peuvent s'expliquer en partie par les représentations dont l'entreprise est l'objet. Selon A. Gonnin-Bolo ${ }^{11}$, le clivage entre la norme professionnelle, qui accepte le monde du travail, et la norme scolaire, fondée sur l'acquisition d'une culture générale, explique pour beaucoup l'état des relations entre les deux entités. Les vieilles querelles n'ont pas disparu: l'école reproche plus ou moins explicitement à l'entreprise sa logique de profit et sa vision court-termiste. La retenue de l'école est sans doute renforcée par les pratiques adoptées par certaines entreprises en période de crise, en particulier dans les opérations de restructuration et de licenciement. À l'inverse, il n'est pas rare d'entendre des responsables d'entreprises reprocher à l'école son manque de sens de la réalité et le manque de professionnalisme des jeunes diplômés. L'école doitelle former pour l'entreprise ? Certainement pas. Elle doit toutefois aider les jeunes à appréhender le monde avec lucidité, à développer leur autonomie et favoriser leur insertion. Quoiqu'on en pense, les élèves de tous âges sont au contact quotidien des entreprises, que ce soit par leur famille, la consommation, la publicité, ou encore les produits pédagogiques. Vouloir protéger l'école en ignorant ces réalités est une utopie. Vouloir organiser les liens et les actions en relation avec les entreprises en gardant à l'esprit les missions et les valeurs de l'école est une nécessité. Pour donner une image, il s'agit de « gérer la distance »...

\section{Les résultats d'une étude exploratoire sur les représentations sociales de l'entreprise}

7 Dans quelle mesure les représentations sociales sur l'entreprise peuvent-elles expliquer l'état des relations école - entreprise -aujourd'hui ? Que peut apporter la connaissance de ces représentations à l'organisation d'actions d'information, d'échange, d'orientation et d'insertion professionnelle? La problématique soulevée nécessite de s'intéresser aux élèves, aux enseignants, aux responsables d'établissements scolaires et aux responsables d'entreprises. Les résultats présentés dans ce papier sont issus d'une enquête réalisée entre janvier 2000 et juin 2001 en trois étapes. La première s'est limitée a des élèves de filières tertiaires (Seconde, Première et Terminale STT, BTS Tertiaires, DPECF, DECF) de lycées de l'Académie de Montpellier ; 434 questionnaires ont été traités. Dans un second temps, l'étude a été étendue à des étudiants de l'IUT de Béziers appartenant aux formations DUT Techniques de Commercialisation et Génie des Télécommunications et Réseaux. 190 questionnaires ont pu être exploités. La forte présence d'étudiants post-bac se justifie par nos interrogations sur l'insertion professionnelle. Dans un troisième temps, 
nous avons interrogé Conseillers Principaux d'Éducation et des professeurs stagiaires ${ }^{12} \mathrm{de}$ toutes disciplines de l'Académie de Montpellier; 160 professeurs et CPE stagiaires appartenant à 20 disciplines différentes ont finalement participé (cf. tableau. 1).

Tableau 1

\begin{tabular}{lc}
\hline L'échantillon d'élèves - étudiants & $\begin{array}{c}\text { Questionnaires } \\
\text { traités }\end{array}$ \\
\hline Classe & 76 \\
\hline Seconde - Option STT & 35 \\
\hline Première STT - Option AAC & 24 \\
\hline Terminale STT & 49 \\
Option ACA & 45 \\
Option CG & 35 \\
Option ACC & 33 \\
\hline BTS Compta - Gestion & 34 \\
\hline BTS Assistant Secr. Trilingue & 28 \\
\hline BTS Action Commerciale & 24 \\
\hline BTS Assistant de Direction & 9 \\
\hline BTS Force de Vente & 17 \\
\hline BTS “ autre " & 25 \\
\hline DPECF & 95 \\
\hline DECF & 95 \\
\hline DUT Techniques de Commercialisation & \\
\hline DUT Génie Télécommunications et Réseaux & \\
\hline
\end{tabular}

L'enquête sera étendue ultérieurement aux responsables d'entreprises, aux responsables d'établissements scolaires, et aux élèves d'autres filières.

Les représentations sociales sur l'entreprise constituent à notre connaissance un thème peu abordé par les chercheurs. L'étude réalisée par l'équipe d'Aix-en-Provence en 2000 auprès d'élèves de Seconde de lycées professionnels et de lycée d'enseignement général constitue donc une référence utile ${ }^{13}$. On mentionnera aussi l'ouvrage de Audigier et Chatel (1987) ${ }^{14}$, et les travaux de Le Merrer et al.(1985) ${ }^{15}$. -L'étude présentée ici doit être considérée comme exploratoire, sa seule ambition étant de fournir des pistes de réflexion et de débat.

Qu'entendons-nous par représentation sociale ? Selon G.N. Fisher (1987), c'est « [...] un processus d'élaboration perceptive et mentale de la réalité qui transforme les objets sociaux en objets symboliques et leur confere un statut cognitif permettant d'appréhender les aspects de la vie ordinaire par un recadrage de nos propres conduites à l'intérieur des interactions - sociales ». Elle induit donc une manière d'organiser notre connaissance de la réalité. Cette connaissance s'élabore à partir de nos codes d'interprétation, culturellement marqués, et constitue un phénomène social en soi. Comme l'expliquent Chazalon et $\mathrm{al}(2001)^{16}$, les représentations sont construites au travers de pratiques quotidiennes et partagées par l'ensemble d'un groupe social, au-delà des particularités individuelles. D'origines diverses (famille, médias, école...) elles forment des construits préalables à l'apprentissage. La formation des représentations sociales s'explique par trois catégories de facteurs : la complexité de l'objet (ici l'entreprise), la diversité des sources d'information et le caractère partiel des informations détenues sur l'objet de la représentation. Sur la base des travaux de Abric (1994), nous avons cherché à identifier les éléments centraux, à savoir le noyau dur des représentations sociales de l'entreprise. Il s'agit de dévoiler les significations 
profondes qu'un groupe, à un moment donné, associe à un objet social, pour comprendre ses perceptions et ses attitudes à l'égard de cet objet.

Le recueil des données été réalisé en une fois, par questionnaire. La taille et la structure de l'échantillon d'élèves - étudiants permet d'apprécier l'évolution des représentations à la fois en fonction du niveau scolaire et de la filière. À cet effet, plusieurs questions d'évocation (ouvertes) ont été intégrées. Il s'agit notamment de citer des mots qui viennent à l'esprit quand on pense à l'entreprise, ou encore de citer spontanément des noms d'entreprises ou des événements d'actualité. Le questionnaire visait également à identifier les perceptions des élèves et professeurs stagiaires vis-à-vis de l'insertion professionnelle et des relations école - entreprise. On gardera à l'esprit lors de la lecture des résultats le fait que seuls des élèves de filières tertiaires ont été interrogés. Pour simplifier la présentation, les résultats "élèves" et "professeurs stagiaires " sont présentés en parallèle. Les divergences sont pointées le cas échéant.

\section{La représentation sociale de l'entreprise : quelles composantes?}

11 À quoi vous fait penser le mot entreprise ? La première question d'évocation permet d'identifier cinq pôles principaux chez les élèves comme chez les professeurs et CPE stagiaires :

- Le pôle « travail - relations de travail » est le plus important. Il regroupe $40 \%$ des réponses exprimées. À l'instar de Chazalon et al. (2001), nous constatons que le travail constitue le cœur des représentations sociales de l'entreprise. Il apparaît en tant qu'activité (emploi, vie active, licenciement, chômage) et en termes de vie au travail (collaboration, rémunération, organisation, ambiance. On note une évocation fréquente du groupe de travail (équipe, communauté) et des contraintes du travail (flexibilité, 35 heures, pointage), ce qui rejoint les études récentes sur la vie au travail.

- Le second pôle concerne « la performance » de l'entreprise. Deux conceptions distinctes se dégagent : la première renvoie à la performance financière, autour des notions de profit, de rentabilité, de productivité et d'argent, alors que la seconde renvoie à la compétitivité " hors coûts », autour des notions de dynamisme, d'innovation, de concurrence, de vente, ou de qualité. Ce pôle apparaît avec une fréquence de l'ordre de $25 \%$.

- Le troisième pôle concerne « les acteurs » de l'entreprise et leur statut : salariés, employeur, hiérarchie. Il représente environ $20 \%$ des réponses. Comme le notent Chazalon et al. (2001), on peut y voir une représentation de l'entreprise moyenne, hiérarchisée, où les fonctions sont clairement dissociées. On notera sur ce point que les syndicats ne sont jamais évoqués par les élèves interrogés.

- Le quatrième pôle évoque « les évolutions de l'entreprise » : informatique, mondialisation, services. Les répondants semblent avoir bien intégré la tertiarisation des entreprises, autour du triptyque « services - clients - vente ». On note que le mot « ouvrier » n'est jamais prononcé.

- Plus marginal, le dernier pôle aborde la « responsabilité de l'entreprise » et « la place de l'entreprise dans la société ». L'entreprise est associée à des mots comme corruption, citoyenneté, respect, jungle. On trouve ici des réponses plus « critiques », plus engagées, qui marquent une prise de recul par rapport à l'entreprise, son image, et son rôle social. Ce pôle représente une quinzaine de répondants sur l'ensemble de l'échantillon.

12 On constate sans véritable surprise la place du travail au cœur des représentations sociales de l'entreprise, à la fois dans sa dimension « emploi » et dans sa dimension «vie 
au travail ». Le travail en entreprise est vu comme un moyen de subsistance et de sécurité matérielle mais aussi comme un moyen d'intégration sociale et une source d'épanouissement pour les élèves. Les événements d'actualité cités par les répondants ne semblent donc pas remettre en cause la valeur et le sens du travail salarié. Les réponses fournies témoignent sur ce point d'une bonne connaissance des évolutions organisationnelles, mais révèlent plusieurs contradictions :

- Si le travail leur apparaît essentiel, les élèves et étudiants interrogés pensent qu'il est plus difficile de trouver un emploi aujourd'hui, et que le travail est souvent plus précaire.

- Le travail est jugé plus valorisant qu'avant mais plus pénible et plus contraignant. Si l'informatisation et la mécanisation ont rendu le travail plus facile aux yeux des répondants, ils considèrent les conditions de travail plus pénibles, surtout en raison des contraintes imposées par la flexibilité.

13 Les attentes vis-à-vis du travail contrastent avec la vision des valeurs des entreprises et de l'état des relations sociales: pour la majorité des élèves comme des étudiants, l'entreprise, ce serait plutôt la loi de la jungle qu'une grande famille, et la recherche du profit le principal moteur de l'entreprise. Les réponses sont plus partagées entre élèves de tertiaire et étudiants de l'IUT en ce qui concerne la façon dont les dirigeants traitent leur personnel: pour les élèves de «tertiaire", les dirigeants sont jugés plutôt « exploiteurs» (score de 3,35/5), alors que les étudiants de l'IUT sont plus indulgents (score de 2,80/5).

\section{La grande entreprise : le modèle dominant}

Les réponses fournies montrent que la représentation spontanée de l'entreprise chez les élèves et les professeurs stagiaires est incontestablement celle de la grande entreprise. Les entreprises les plus citées sont des sociétés ou des groupes de taille nationale ou mondiale : IBM, Carrefour, Renault, Coca-Cola, EDF-GDF, France Télécoms, SNCF, Total, Cora, Auchan, Peugeot, Microsoft, etc. Les secteurs les plus évoqués sont l'industrie traditionnelle (automobile), l'énergie, la distribution, l'informatique et la communication (téléphonie surtout). Si on note une part non négligeable d'entreprises implantées localement ( $40 \%$ des réponses environ), ce sont surtout des filiales, établissements ou sites de groupes et enseignes de taille nationale ou internationale. Les PME-PMI locales indépendantes n'occupent qu'une place limitée, alors qu'elles représentent plus de $90 \%$ de l'économie française. Dans les mots cités spontanément, le mot «PME » n'apparaît qu'une seule fois... Dans le même ordre d'idée, on constate que bon nombre de noms d'entreprises renvoient en même temps à des marques (IBM, Coca-Cola, Nike, Perrier, Carrefour, Danone), ce qui démontre l'importance des expériences de consommation dans la formation des représentations. On mesure ici l'impact des campagnes publicitaires des grandes firmes sur les élèves et leur champ de référence...

Dans l'actualité, ce sont surtout les événements très médiatisés, qui attirent l'attention. L'entreprise est interpellée sur des questions de citoyenneté, de responsabilité écologique ou sociale: Total-Fina avec l'Erika (marée noire), Moulinex et Michelin avec leurs licenciements représentent à eux seuls près de $70 \%$ des réponses. Les autres événements cités sont des opérations de fusion (AOL - Time Warner) ou de rachat (France Télécom Orange). Dans de très rares cas sont évoqués des événements comme la sortie d'un nouveau produit, une innovation sociale, a fortioridans une entreprise locale. 


\section{Les attentes des élèves : quel portrait de l'entreprise idéale?} élèves (cf. tableau. 2, ci-dessous) ? Leurs réponses sont claires: pour 2/3 des répondants, l'entreprise « idéale » est une entreprise de taille moyenne (50 à 500 salariés) ou petite (10 à 50). La grande entreprise n'obtient que $19 \%$ des suffrages. En ce qui concerne le secteur, il s'agit en grande majorité du tertiaire (privé ou public), ce qui s'explique par la formation des répondants: comptabilité, commercial, banque, administration, informatique, télécommunications, tourisme, grande distribution, etc. L'industrie ou l'artisanat n'obtiennent que $5 \%$ des réponses. Les élèves expriment des attentes précises vis-à-vis du travail et des relations sociales: ils recherchent une entreprise qui favorise la coopération, la participation du personnel, le travail en équipe, et qui s'appuie sur des valeurs plus sociales que financières ${ }^{17}$. Concernant le statut les réponses sont plus partagées : pour les élèves de lycée, le privé et le public sont à égalité. Le statut associatif représente $5 \%$ des réponses. Pour les étudiants de l'IUT, c'est le privé qui l'emporte largement (2/3 des réponses) et on note chez les étudiants de Techniques de Commercialisation un attrait croissant pour le privé entre la première et la seconde année.

Tableau 2

\begin{tabular}{|llllllll|}
\hline Portrait de l'entreprise « idéale » pour les élèves \\
\hline Entreprise très jeune & 1 & 2 & 3 & 4 & 5 & Entreprise expérimentée \\
\hline Ambiance de compétition & 1 & 2 & 3 & 4 & 5 & Ambiance de coopération \\
\hline Direction autoritaire & 1 & 2 & 3 & 4 & 5 & Direction participative \\
\hline Travail en équipe & 1 & 2 & 3 & 4 & 5 & Travail individuel \\
\hline Très forte autonomie & 1 & 2 & 3 & 4 & 5 & Travail très encadré \\
\hline Culture de dynamisme & 1 & 2 & 3 & 4 & 5 & Culture de stabilité \\
\hline Valeurs sociales & 1 & 2 & 3 & 4 & 5 & Valeurs financières \\
\hline
\end{tabular}

17 Ce portrait fait clairement apparaitre la PME comme l'entreprise idéale pour les élèves et étudiants interrogés. Il confirme l'importance attachée au travail - dans ses dimensions organisationnelle, sociale et culturelle - et aux valeurs de l'entreprise. Les "affaires» récentes évoquées précédemment ont certainement contribué à renforcer cette position. On constate toutefois un paradoxe dans les réponses obtenues auprès des élèves de lycée : comment concilier petite taille et statut public?

\section{L'insertion professionnelle et les relations école entreprise}

18 La problématique abordant le thème de l'insertion professionnelle, nous avons tenté d'identifier la perception des élèves, professeurs et CPE stagiaires, à propos des critères de recrutement privilégiés par les responsables d'entreprises et à propos des dispositifs de rapprochement ou de "partenariat " école - entreprise. Sur ces aspects, on relève un certain nombre de divergences entre élèves et professeurs stagiaires, mais aussi entre élèves. 


\section{Le point de vue des élèves}

19 Concernant les critères « objectifs » de recrutement, les réponses sont homogènes: les diplômes, l'expérience professionnelle, puis la maîtrise des langues et de l'informatique sont considérés comme des critères essentiels pour les dirigeants. Arrivent ensuite des critères comme le physique, la mobilité géographique, les "relations ", ou l'âge. Ce sont donc des critères « classiques » de recrutement qui sont jugés les plus déterminants. On notera qu'un nombre non négligeable d'élèves pense que le physique et les relations (le " piston») interviennent dans les choix de recrutement. Le sexe et la situation familiale sont jugés peu importants. Au niveau des qualités recherchées, les élèves de lycée et les étudiants de l'IUT n'ont pas le même point de vue : pour les premiers, les entreprises sont surtout sensibles aux connaissances techniques et à la maîtrise de l'informatique. Vient ensuite le dynamisme. Pour les étudiants de l'IUT, le dynamisme et la communication orale arrivent en tête des qualités jugées prioritaires, même si les savoirs techniques suivent de près. On remarque donc le poids des savoirs et des savoir-faire dans la représentation que se font les élèves des critères de recrutement: les connaissances techniques, la maîtrise de l'outil informatique, la communication orale constituent selon eux les qualités à développer. On notera pour l'anecdote que les qualités comme le courage, l'esprit d'initiative ou l'esprit critique arrivent très loin derrière les autres critères, et sont donc jugés peu importants dans les choix d'embaucher ${ }^{18}$. Ces réponses montrent que la représentation du métier de gestionnaire (les élèves interrogés sont, de futurs gestionnaires) reste technique, même si dynamisme et communication orale occupent une place significative. En revanche, la faible fréquence pour l'esprit critique donne à réfléchir...

\section{Le point de vue des professeurs et CPE stagiaires}

Le point de vue des professeurs stagiaires est sensiblement différent de celui des élèves. $\mathrm{Si}$ ces derniers jugent les savoirs et savoir-faire prioritaires dans les choix des recruteurs, les professeurs et CPE stagiaires mettent davantage l'accent sur les savoir-être : pour eux, ce sont surtout des qualités comme l'adaptabilité, la mobilité, la capacité de travail, le dynamisme, ou encore l'esprit d'initiative qui sont essentiels pour intégrer l'entreprise. Il est difficile d'interpréter le décalage entre les élèves et les professeurs stagiaires, sachant qu'ils n'appartiennent pas aux mêmes formations. Pour autant, il serait utile d'identifier les mécanismes aidant les élèves à se "rassurer " quant à leur employabilité et les conduisant à se focaliser sur leurs connaissances techniques, alors que leurs enseignants insistent fréquemment sur l'importance des savoirs et savoir-être... Une réflexion est également à mener au niveau des méthodes pédagogiques pouvant favoriser le développement des savoir-être jugés prioritaires par les enseignants.

\section{Comment améliorer les relations école - entreprise, la faute à qui ?}

21 Concernant les relations école - entreprise, les réponses des élèves sont très partagées (cf. tableau. 3, ci-dessous) ; les réponses sont jugées ni bonnes, ni mauvaises. Les critiques des élèves s'adressent aux deux parties: à l'école, ils reprochent le plus souvent un manque d'ouverture et d'informations sur le monde des entreprises, un manque de responsabilité, de motivation et de professionnalisme. Ils veulent plus de stages, plus de 
temps pour aller en entreprise, plus de sorties et de visites en entreprise, et, de façon plus générale, plus de communication entre école et entreprise. Les critiques faites aux entreprises vont dans le même sens: ils souhaiteraient plus de stages, plus de disponibilité, plus d'ouverture à l'égard de jeunes sans expérience. Il ne s'agit pas de créer de nouveaux dispositifs mais de s'appuyer sur les dispositifs classiques pour développer l'ouverture aux jeunes. Dans le même ordre d'idée, le côté psychologique de l'accueil de stagiaires est apparu déterminant : les répondants demandent aux entreprises plus de confiance, plus de considération, plus d'écoute et plus de reconnaissance envers les stagiaires et les jeunes embauchés.

Tableau 3

\begin{tabular}{lc}
\hline Les souhaits des élèves pour améliorer les relations école-entreprise \\
\hline Souhaits & \% réponses \\
\hline Du côté des entreprises & 32 \\
Plus d'ouverture envers l'école & 27 \\
Plus de stages & 22 \\
Plus de considération, de confiance & 20 \\
Plus d'intégration, d'encadrement & 50 \\
\hline Du côté de l'école & 34 \\
Plus d'ouverture vers l'entreprise & 15 \\
\hline Plus de stages & \\
\hline Plus d'implication des enseignants
\end{tabular}

Les CPE et professeurs stagiaires sont plus pessimistes que les élèves, puisque $2 / 3$ d'entre eux les jugent mauvaises ou très mauvaises. À noter d'ailleurs que 80 \% d'entre eux a déjà eu une expérience en entreprise (stage, emploi saisonnier). Pour faire évoluer cet état de fait, quelles actions sont-ils prêts à soutenir? Comme les élèves, ils jugent prioritaires les actions d'ouverture vers l'entreprise (contacts, visites, conférences...), et les stages en entreprise ( $c f$. tableau. 4 , ci-dessous). Seuls $4 \%$ des répondants évoquent l'aide à l'orientation, ce qui tend à démontrer que le lien entre la découverte des entreprises et l'aide à l'orientation n'est pas perçu dans la grande majorité des cas...

Tableau 4

\begin{tabular}{lc}
\hline Les actions que les professeurs stagiaires sont prêts à soutenir \\
\hline Actions proposées & $\%$ réponses \\
\hline Développer des ouvertures (visites, partenariats, contérences...) & 63 \\
\hline Développer les stages & 18 \\
\hline Favoriser les recherches pour les cours & 14 \\
\hline Favoriser les recherches pour l'orientation & 4 \\
\hline
\end{tabular}

Le décalage entre les logiques de l'école et de l'entreprise (y compris pour des formations type BTS) et la diversité des types de stages en entreprise imposent une préparation et un encadrement importants des échanges école - entreprise, ne serait-ce que pour élaborer une représentation commune de ce qu'on attend de l'expérience et du stagiaire. Se pose donc assez rapidement la question des moyens d'encadrement et du temps donné aux formateurs pour ces missions. 


\section{Enseignements et prolongements pratiques de la recherche}

24 Si elle comporte plusieurs limites méthodologiques, l'enquête présentée au point précédent contribue à identifier plusieurs tendances qui donnent des pistes d'actions pour l'organisation des relations école - entreprise :

- Les connaissances très aléatoires sur le milieu économique et social : les représentations très centrées sur la grande entreprise (moins de $5 \%$ en termes de nombre d'entreprises dans l'économie française) suggèrent de développer une information bien structurée intégrant les PME, qui constituent la majorité des entreprises françaises (environ $95 \%$ ). Cette information doit être complétée par la découverte des métiers et de la diversité des parcours professionnels. Cet aspect entre dans le cadre de l'éducation à l'orientation.

- Les appréciations contrastées sur les entreprises et leurs pratiques : par leur essence même elles trouvent « naturellement » leur place dans des dispositifs faisant une large place aux débats - tel l'ECJS - ou peuvent servir de motif pour découvrir l'entreprise, de point d'appui dans les travaux pluridisciplinaires menés en groupes restreints - tels les IDD (Itinéraire de Découverte) et les TPE (Travaux Personnels Encadrés).

- Les souhaits exprimés pour améliorer les relations école - entreprise : l'ouverture à l'entreprise de façon ponctuelle (visite, conférence...) est plébiscitée de façon assez large par les répondants, qui expriment aussi des attentes en ce qui concerne le contenu, l'organisation et le suivi des stages en entreprise.

Ces résultats encouragent d'une part à analyser de manière approfondie les initiatives des établissements en matière de partenariat pour en dégager une sorte de "cahier de charges » à suivre par l'enseignant et sa classe pour préparer, organiser, cadrer et exploiter ces ressources externes. D'autre part, il convient de faire mieux connaître les dispositifs de stages qui existent depuis longtemps dans les séries professionnelles (lycée professionnel ${ }^{19}$, STS, IUT...) et de mener une réflexion sur la manière de les adapter à d'autres niveaux, en particulier en classe de $3^{\text {e }}$ des collèges.

\section{L'expérimentation menée à l'IUFM de l'académie de Montpellier}

L'expérimentation: La formation des professeurs stagiaires de toutes disciplines et CPE stagiaires dans le module "ouverture au monde de l'entreprise ». Elle est réalisée à l'IUFM de l'Académie de Montpellier, en formation initiale dispensée auprès des professeurs stagiaires et CPE stagiaires du second degré (environ 800 stagiaires répartis dans 32 groupes sur les divers sites de l'IUFM pour l'année universitaire 2002-2003).

\section{Les objectifs du module}

27 Il s'agit d'abord de sensibiliser ${ }^{20}$ les futurs enseignants et CPE à leurs "nouvelles » missions (voir section 1). Ensuite il s'agit de faire réfléchir aux actions qu'ils peuvent mener, en utilisant la connaissance des entreprises dans les différents projets à caractère pluridisciplinaire auxquels leur participation est requise (IDD, TPE, ECJS) ou plus simplement dans les projets pédagogiques qu'ils montent avec leur classe dans le cadre de leur discipline. 
Le projet d'établissement et le projet de l'élève constituent le contexte de cette réflexion. La connaissance des entreprises (ou plus modestement l'ouverture au monde des entreprises ${ }^{21}$ ) est dès lors un "levier ", un outil auquel il convient de faire appel pour essayer d'atteindre les objectifs décrits ci-dessus. Outre des témoignages et des échanges, le module a également pour but de transmettre des ressources ${ }^{22}$ utilisables par le professeur pour sa propre formation. Il s'appuie sur un partenariat avec différentes associations et groupements professionnels: la Chambre des Métiers de l'Hérault, le Centre des Jeunes Dirigeants, la Fédération Générale du Bâtiment et le Comider ${ }^{23}$. Il est donc un moment de rencontre entre deux mondes qui se côtoient en général assez peu.

\section{Le projet d'établissement ${ }^{24}$ ?}

C'est un projet pédagogique et éducatif. Il est l'expression de la politique qu'un établissement (école, collège, lycée) choisit de conduire à moyen terme en conciliant l'obligation de respect des objectifs et des programmes nationaux et la nécessité de s'adapter d'une part aux élèves et d'autre part aux spécificités d'une réalité locale. Les partenaires $\mathrm{du}$ projet d'établissement sont non seulement les responsables de l'établissement mais encore les enseignants, les conseillers d'éducation, le personnel ATOS, les parents d'élèves et les élèves.

\section{Le projet de l'élève ${ }^{25}$ ?}

30 C'est l'expression par l'élève d'objectifs qui se déterminent par la mise en concordance de ses envies et de ses résultats. Ces objectifs s'affinent pendant la scolarité; ce qui est important c'est la modification d'attitude, c'est la démarche qui permet d'avancer avec les représentations qu'il a de son environnement familier ou plus lointain, c'est le développement de son autonomie.

\section{Le dispositif de formation (une journée de 6 heures)}

Les 2 parties (matin et après-midi) ont comme dénominateur commun les relations école - entreprise.

La première partie est orientée vers une réflexion et un échange de vues avec des professionnels venus témoigner de leur expérience professionnelle, de leur parcours et des relations qu'ils entretiennent (ou souhaitent entretenir) avec l'école. C'est aussi l'occasion de développer l'information sur les entreprises, les emplois, les milieux professionnels, d'appréhender les évolutions des organisations et du travail pour guider et accompagner les élèves dans la construction de parcours scolaires et professionnels à travers une démarche éducative active et cohérente. Cette "ouverture" passe essentiellement par la connaissance de la diversité des entreprises (taille, statut, activité, culture, organisation du travail, critères d'embauche) et une réflexion sur la dimension humaine de l'entreprise, notamment sous l'angle des savoir-être.

La deuxième partie se veut très concrète en proposant aux stagiaires de travailler par petits groupes en ateliers sur des actions à mener dans leurs [futures] classes, seuls ou dans un cadre pluridisciplinaire. Différentes ressources leur sont proposées :

- des exemples réels de projets menés par des établissements en partenariat avec les entreprises ; 
- une information sur les mesures actuelles telles que les «Itinéraires de découverte ${ }^{26}$ » au collège ; les « $\mathrm{TPE}^{27}$ » au lycée ; les « $\mathrm{PPCP}$ » au lycée professionnel ${ }^{28}$; les « $\mathrm{AMP}^{29}$ » dans les séries technologiques tertiaires ;

- La présentation des stages de découverte de l'entreprise (au niveau des collèges et des lycées généraux et technologiques) et les stages de formation (en alternance ou non au niveau des lycées professionnels ${ }^{30}$ et dans les voies de formation post-bac : supérieur court/long).

\section{Conclusion}

L'information et la découverte du monde des entreprises doivent être développées aux différents niveaux du système scolaire. Il s'agit à notre sens de créer chez l'élève une culture et une pratique minimale des entreprises et des métiers qui puisse l'aider dans ses choix d'orientation et d'insertion professionnelle, et qui l'amène à réfléchir aux compétences à développer par rapport à la vie active. Rappelons que notre idée n'est pas celle d'un partenariat systématique entre école et entreprise, ni celle d'une instrumentation de l'école au service de l'entreprise. Chacune a ses priorités, ses valeurs, ses méthodes. Toutefois, nous croyons qu'entretenir des mythes, des a priori ou des caricatures sur le monde ne peut pas aider les élèves à s'y intégrer en tant qu'individus...

\section{BIBLIOGRAPHIE}

ABRIC J.C. : Coopération, compétition et représentations sociales, Cousset, Delval, 1987.

ABRIC J.C. : « Les représentations sociales : aspects théoriques », in ABRIC J.C. (Éd.), Pratiques sociales et représentations, Paris, PUF, 1994.

AUDIGIER et CHATEL : Entreprises et représentations, Paris, INRP, 1987.

BEITONE A., DECUGIS M. et LEGARDEZ A. :Enseigner les sciences économiques et sociales, Paris,A. Colin, 1995.

CHAZALON D., GAVINI A.M., LEBATTEUX N. et LEGARDEZ A., : « Les représentations sociales de l'entreprise chez les élèves de seconde », in Revue Tertiaire, février 2001.

GIONNIN-BOLO A. : Ecole et entreprise, des partenariats en marche, Paris, INRP, 1998.

LE MERRER, PETIT, MALBURET, SCHERRER et SIMON : Documents pour l'enseignement économique et social, $\mathrm{N}^{\circ} 61$, octobre, 1985.

LOUCHE C. et MOLINIER P. : Sens et représentation du travail chez des télétravailleurs et des travailleurs classiques, Laboratoire de Psychologie du Travail, Université Montpellier III, 2002.

PELPEL P. et TROJET V. : Histoire de l'enseignement technique, Paris, Hachette, 1993.

TROJET V. : « Les élèves de lycées professionnels et des filières technologiques de lycée », in Communication aux Journées IUFM des Formateurs Economie-Gestion, IUFM d'Antony, 1998. 


\section{NOTES}

1. Voir B.O. $\mathrm{N}^{\circ} 15 \mathrm{du} 11-4-2002$.

2. Formation dite « PLC2 ».

3. Obligatoire pour les futurs professeurs d'enseignement technologique et professionnel, les CPE et les futurs professeurs d'enseignement général des lycées professionnels, le stage en entreprise peut également concerner, si le plan de formation le prévoit, les professeurs stagiaires des filières d'enseignement général.

4. Le droit au conseil en orientation et à l'information sur les enseignements et les professions est défini par la loi d'orientation du 10 juillet 1989 (art. 8. Sur la mise en œuvre de l'éducation à l'orientation dans les lycées, voir circulaire du 01/10/1996). Pour l'expérimentation dans les collèges, voir circulaire du 31/07/1996.

5. Accor, EDF, PSA, La Poste, et bientôt la SNCF.

6. Les Ingénieurs Pour l'Ecole sont des cadres de grandes entreprises détachés auprès des rectorats.

7. Des expériences existent déjà : dans l'Académie de Toulouse, des mini-stages en entreprise, organisés en collaboration avec l'UIMM, sont proposés à des enseignants de diverses disciplines.

8. Elles s'appuient en particulier sur les stages.

9. Suppl. de la Lettre de l'Education, $\mathrm{N}^{\circ} 289$, « Non plus ennemies, mais pas encore partenaires », décembre 1999.

10. GONNIN-BOLO A. : Ecole et entreprise, des partenariats en marche. Paris, INRP, $140 \mathrm{p}$.

11. Op. cit.

12. Enseignant ayant réussi à un concours de recrutement de l'Education Nationale et en formation pendant un an en vue de sa titularisation.

13. CHAZALON et al : «Les représentations sociales de l'entreprise chez les élèves de seconde », in Revue Tertiaire, février 2001.

14. AUDIGIER et CHATEL : Entreprises et représentations. Paris, INRP, 1987.

15. LE MERRER et al. : Documents pour l'enseignement économique et social, $\mathrm{N}^{\circ} 61$, octobre 1985.

16. Op. cit.

17. Le test de Fisher tend à montrer que ce sont surtout les élèves de Seconde, Première et Terminale qui privilégient les valeurs sociales, alors que les élèves de BTS semblent plus sensibles aux valeurs financières de l'entreprise. L'ensemble de ces résultats confirme l'importance accordée aux relations sociales, à l'ambiance de travail, et à la culture organisationnelle.

18. Le test de Fisher ne révèle pas de différence significative selon la profession des parents, que ce soit pour les critères « objectifs » ou les qualités personnelles.

19. Stage en BEP, période de formation en entreprise en Bac Professionnel.

20. L'expérience menée depuis novembre 2002, dans l'académie de Montpellier, montre qu'il s'agit avant tout d'une information car les stagiaires méconnaissent majoritairement leurs missions en dehors de leur discipline.

21. D'où l'intitulé de la formation.

22. Sous forme multimédia.

23. Comité pour le développement de l'économie régionale (association).

24. Extraits de la loi d'orientation de 1989.

25. Extraits de la cassette vidéo, le projet personnel de l'élève, Montpellier, CRDP de l'Académie de Montpellier, 1996.

26. B.O No 31 du 29/08/2002.

27. Travaux Personnels Encadrés - B.O N 29 du 27/07/2000.

28. Voir B.O N² 25 du 29/06/2000. 
29. Activités en Milieu professionnel.

30. Stage en entreprise en BEP et Période de formation en entreprise en Bac Professionnel - B.O $\mathrm{N}^{\circ} 25 \mathrm{du} 29 / 06 / 2000$.

\section{RÉSUMÉS}

Ce papier présente de façon synthétique les résultats d'une étude exploratoire des représentations sociales sur l'entreprise réalisée en 2000-2001 dans l'académie de Montpellier auprès d'élèves, de professeurs et de CPE stagiaires. Cette recherche a débouché en 2001-2002 sur la proposition d'un projet innovant et l'expérimentation d'un module de formation destiné aux professeurs et CPE stagiaires de l'académie.

This research paper presents the synthesis of the resuits of an exploratory study on social representations about corporations/businesses, conducted in 2000-2001 in the Montpellier "académie", the public polled consisting of students, teachers and school counsellor ("CPE") trainees. The result of this research was in 2001-2002 a proposal being made for an innovative action project and the experimentation of a training module specially conceived for teachers and school counsellor trainees in the Montpellier "académie"

\section{INDEX}

Mots-clés : connaissance des entreprises, relation école - entreprise, représentation sociale Keywords : knowledge of corporations/companies, knowledge of the business world, schoolbusiness relation, social representation

\section{AUTEURS}

\section{ANNE-MARIE MANVILLE}

PRAG, IUFM de Montpellier

\section{CLAUDE FABRE}

Maître de conférences, IUFM de Montpellier 